\title{
Supercontinuum generation in fibers infiltrated with liquid crystals
}

\author{
Rasmussen, Per Dalgaard; Bang, Ole; Lægsgaard, Jesper; Rottwitt, Karsten
}

Published in:

International Conference on Transparent Optical Networks, 2006

Link to article, DOI:

10.1109/ICTON.2006.248321

Publication date:

2006

Document Version

Publisher's PDF, also known as Version of record

Link back to DTU Orbit

Citation (APA):

Rasmussen, P. D., Bang, O., Lægsgaard, J., \& Rottwitt, K. (2006). Supercontinuum generation in fibers infiltrated with liquid crystals. In International Conference on Transparent Optical Networks, 2006 (Vol. 2, pp. Mo.D2.3). IEEE. https://doi.org/10.1109/ICTON.2006.248321

\section{General rights}

Copyright and moral rights for the publications made accessible in the public portal are retained by the authors and/or other copyright owners and it is a condition of accessing publications that users recognise and abide by the legal requirements associated with these rights.

- Users may download and print one copy of any publication from the public portal for the purpose of private study or research.

- You may not further distribute the material or use it for any profit-making activity or commercial gain

- You may freely distribute the URL identifying the publication in the public portal 


\title{
Supercontinuum Generation in Fibers Infiltrated with Liquid Crystals
}

\author{
Per Dalgaard Rasmussen, Ole Bang, Jesper Lagsgaard, and Karsten Rottwitt \\ $C O M \cdot D T U$ Department of Communications, Optics \& Materials \\ Technical University of Denmark, Ørsteds Plads 345V, DK-2800 Kgs. Lyngby, Denmark \\ Tel: (+45) 45256357,e-mail:pdr@com.dtu.dk
}

\begin{abstract}
Supercontinuum generation in a capillary tube infiltrated with a nematic liquid crystal is investigated theoretically in the near infrared region. A liquid crystal with a high electronic nonlinearity is chosen, which makes it possible to generate $100 \mathrm{~nm}$ wide supercontinua using $10 \mathrm{ps}$ pulses with peak power $1.5 \mathrm{~kW}$ in a $10 \mathrm{~cm}$ long waveguide. The possibility of tuning the spectrum of the generated supercontinuum by changing the dispersion of the waveguide is also considered. It is found that the broadening of the spectrum in both the normal and anomalous regime is mainly due to self phase modulation, and therefore the dispersion of the waveguide is only of minor importance. The tuning of the dispersion is achieved by varying the temperature of the liquid crystal inside the capillary.
\end{abstract}

Keywords: nonlinear optics, liquid crystals, supercontinuum generation.

\section{INTRODUCTION}

Recently the possibility of making tunable optical devices based on liquid crystals (LCs) has attracted much attention [1]. Such devices can be realized, because the optical properties of LCs can be varied by changing the temperature or applying an external electric field. Together with the unique properties of photonic crystal fibers (PCFs) such as endlessly singlemode, and zero dispersion wavelengths in the visible spectrum, optical devices based on PCFs and LCs hold ongoing promise for potential applications in photonics. Along with these tunable properties, LCs also possess high nonlinearities. When using LCs in nonlinear optics it is often the reorientational nonlinearity of the LC that is being exploited, since this nonlinearity can be several orders of magnitude larger than in silica [2]. But since the time it takes to reorient the LC molecules is of the order of microseconds it cannot be used for supercontinuum generation (SCG) with short pulses, where the pulses are of the order of picoseconds or femtoseconds. For such short pulses it is the fast electronic nonlinearity of the material that is responsible for SCG. It turns out that this fast electronic nonlinearity for many LCs is about two orders of magnitude larger than in silica. Often SCG has been considered at visible wavelengths, which can be used for optical coherence tomography. But also at the telecommunication wavelengths around $1550 \mathrm{~nm} \mathrm{SCG}$ also has possible applications, since it can be used to produce short pulses at multiple wavelengths simultaneously, which can be used for WDM communication systems [3].

In the present work we propose a supercontinuum light source in the near infrared spectrum consisting of a capillary silica tube infiltrated with LC. The high electronic nonlinearity of the LC allows us to generate $100 \mathrm{~nm}$ wide spectra from $10 \mathrm{ps}$ pulses with peak power $1.5 \mathrm{~kW}$. The temperature dependence of the refractive index of the LC allows us to change the dispersion profile of the device, and thereby investigate how the generated spectra are modified.

\section{THEORY}

In this work we use data for the LC 5CB, since a large amount of experimental data for the refractive index as a function of temperature and wavelength exists. At room temperature $5 \mathrm{CB}$ is in the nematic phase, and is therefore anisotropic. Usually the experimental data for the ordinary and extraordinary refractive index as a function of wavelength is fitted to a Cauchy polynomial, i.e.

$$
\begin{aligned}
& n_{\perp}=A_{1}^{\perp}+\frac{A_{2}^{\perp}}{\lambda^{2}}+\frac{A_{3}^{\perp}}{\lambda^{4}}, \\
& n_{\|}=A_{1}^{\|}+\frac{A_{2}^{\|}}{\lambda^{2}}+\frac{A_{3}^{\|}}{\lambda^{4}} .
\end{aligned}
$$

Where the indices $\perp$ and $\|$ denotes the ordinary and extraordinary refractive index of the nematic LC. The coefficients $\mathrm{A}^{\perp, \|_{i}}$ as reported by Li et al. [4] are given in Table 1. For the material dispersion of silica we use the Sellmeier curve

$$
n_{\mathrm{SiO} 2}=\sqrt{1+\sum_{j=1}^{3} \frac{a_{j} \lambda^{2}}{\lambda^{2}-b_{j}}},
$$


where $a_{i}$ and $b_{i}$ are constants. Here we use the values given in Table 1 as reported by Okamoto [5].

Table 1. Parameters for Cauchy and Sellmeier polynomials for 5CB and silica given in equations

(1-2) and (3). The non-dimensionless parameters are all given in units of $\mu m^{2}$ or $_{\mu m^{4}}$.

\begin{tabular}{|c|c|c|c|c|c|c|}
\hline$i$ & $A_{i}^{\perp}\left(25^{\circ} \mathrm{C}\right)$ & $A_{i}^{\perp}\left(25^{\circ} \mathrm{C}\right)$ & $A_{i}^{\perp}\left(34.7^{\circ} \mathrm{C}\right)$ & $A_{i}^{\perp}\left(34.7^{\circ} \mathrm{C}\right)$ & $a_{i}$ & $b_{i}$ \\
\hline 1 & 1.51866 & 1.67946 & 1.53028 & 1.64304 & 0.6965325 & $4.368309 \cdot 10^{-3}$ \\
\hline 2 & 0.00163 & 0.00482 & 0.00183 & 0.00380 & 0.4083099 & $1.394999 \cdot 10^{-2}$ \\
\hline 3 & 0.00114 & 0.00273 & 0.00135 & 0.00217 & 0.8968766 & 97.93399 \\
\hline
\end{tabular}

The fast electronic nonlinearity of this LC is measured in terms of the intensity dependent refractive index $n_{2}$. For $5 \mathrm{CB} n_{2, \perp}=1.9 \cdot 10^{-} 18 \mathrm{~m}^{2} / \mathrm{W}$ and $n_{2, \|}=2.5 \cdot 10^{-18} \mathrm{~m}^{2} / \mathrm{W}$, these values have been measured at $532 \mathrm{~nm}$ for $32 \mathrm{ps}$ pulses [6]. Compared to silica fibers these values are about two orders of magnitude larger $\left(n_{2, \mathrm{SiO} 2}=2.4 \cdot 10^{-20} \mathrm{~m}^{2} / \mathrm{W}\right)$. Our waveguide design consists of a circular capillary tube infiltrated with a nematic LC. We assume that the director of the LC aligns parallel with the cylinder axis everywhere in the tube as illustrated in Fig. 1, hence the dielectric tensor in the LC region is given by $\varepsilon=\operatorname{diag}\left(n_{\perp}^{2}, n_{\perp}^{2}, n_{\|}^{2}\right)$.

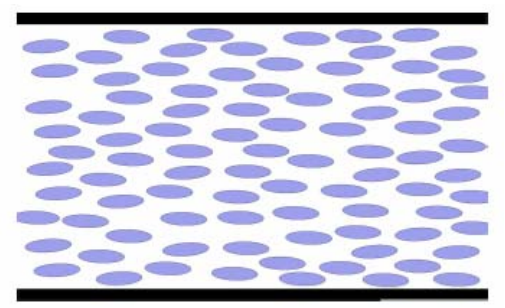

Figure 1. Schematic illustration of the waveguide. The rod-shaped LC-molecules are assumed to be oriented parallel to the axis of the waveguide everywhere inside the capillary.

Since the ordinary index of $5 \mathrm{CB}$ is higher than the index of silica, light can be guided through total internal reflection. The dispersion relation for this step index fiber with an anisotropic core can be found analytically, and is given by [7]

$$
\left[\frac{J_{l}^{\prime}(U)}{U J_{l}(U)}+\frac{K_{l}{ }^{\prime}(W)}{W K_{l}(W)}\right]\left[\frac{\kappa_{c o} J_{l}^{\prime}\left(\kappa_{c o} U\right)}{U J_{l}\left(\kappa_{c o} U\right)}+\frac{1}{W}\left(\frac{n_{c l}}{n_{c o, \perp}}\right)^{2} \frac{K_{l}{ }^{\prime}(W)}{W K_{l}(W)}\right]=\left(\frac{l \beta}{k n_{c o, \perp}}\right)^{2}\left(\frac{V}{U W}\right)^{4},
$$

where

$$
\begin{gathered}
\kappa_{c o}=\frac{n_{c o, \|}}{n_{c o, \perp}}, \\
U=a \sqrt{\left(k n_{c o, \perp}\right)^{2}-\beta^{2}}, \\
W=a \sqrt{\beta^{2}-\left(k n_{c o, \perp}\right)^{2}}, \\
V=\sqrt{U^{2}+W^{2}} .
\end{gathered}
$$

$J_{l}$ is the $l$ 'th order Bessel function, while $K_{l}$ is the $l$ 'th order modified Bessel function. $a$ is the inner radius of the capillary tube, $\beta$ is the propagation constant, and $k$ is the vacuum wavenumber. The criterium for single mode operation is $V<2.405$, i.e. the same as the criterium for an isotropic step index fiber. In this work we only consider the fundamental $\mathrm{HE}_{11}$-mode. The dispersion profile for the waveguide is found by solving (4) numerically, this gives us $\beta$ as a function of $k$. The dispersion is then given by

$$
D=-\frac{k^{2}}{2 \pi c} \frac{d^{2} \beta}{d k^{2}} .
$$

The differentiation of the propagation constant $\beta$ with respect to the wavenumber $k$ is performed using a second order finite difference approach. The material dispersion is included by using the values of the refractive indices as given by equations (1-2) and (3).

To model the propagation of short pulses in this waveguide we use the Nonlinear Schrödinger Equation (NLS) where we neglect losses. The only nonlinear effect we include is self phase modulation, this 
approximation holds as long as the pulses are much longer than $1 \mathrm{ps}$ [8], hence the propagation equation for the envelope of the optical field $A(z, t)$ is

$$
\frac{\partial A}{\partial Z}=i \sum_{n \geq 2} \frac{i^{n} \beta_{n}}{n !} \frac{\partial^{n} A}{\partial t^{n}}+i \gamma|A|^{2} A,
$$

where the nonlinear coefficient is defined by $\gamma=n_{2} \omega_{0} /\left(c A_{\text {eff }}\right)$. It has been assumed that the electric field can be written in the form $E=A(z, t) F(x, y) \exp \left(i\left[\beta z-\omega_{0} t\right]\right) \mathbf{x}$, hence we assume that the light is linearly polarized along $x$. $\omega_{0}=2 \pi c / \lambda$ is the frequency of the pump, $\beta_{n}$ are the coefficients of the Taylor expansion of $\beta(\omega)$ at the pump frequency, i.e. $\beta_{n}=\left(\frac{d^{n} \beta}{d \omega^{n}}\right)_{\omega=\omega_{0}} . A_{\text {eff }}$ is the effective area of the mode we consider (here the $\mathrm{HE}_{11}$-mode, which is almost purely transversal), and is given by

$$
A_{e f f}=\frac{\left(\iint|F(x, y)|^{2} d x d y\right)^{2}}{\iint|F(x, y)|^{4} d x d y} .
$$

In the following we find the dispersion parameters $\beta_{n}$ for different temperatures for a constant pump wavelength, and then calculate the generated spectrum using equation (10).

\section{RESULTS}

The dispersion as a function of vacuum wavelength for the waveguide design has been found for different radii of the capillary tube at $25^{\circ} \mathrm{C}$ and at $34.7^{\circ} \mathrm{C}$. The results are shown in Fig. 2 .
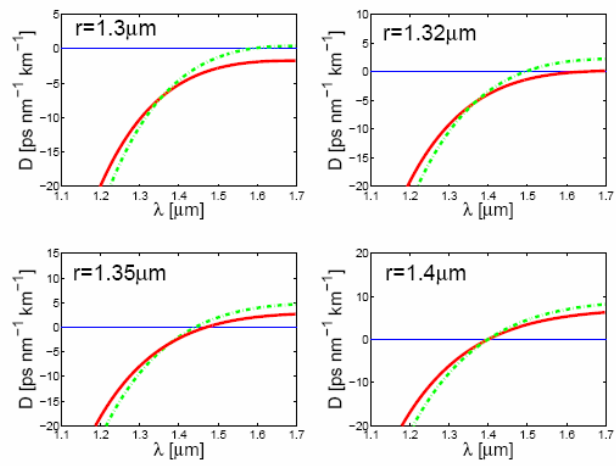

Figure 2. Chromatic dispersion for different radii of the capillary tube infiltrated with $5 C B$ at $25^{\circ} \mathrm{C}$ and at $34.7^{\circ} \mathrm{C}$. Solid lines are for $25^{\circ} \mathrm{C}$ and the dash-dotted lines are for $34.7^{\circ} \mathrm{C}$.

We see that the waveguide can have both normal and anomalous dispersion, depending on the wavelength and temperature. The dispersion of the waveguide with $r=1.3 \mu \mathrm{m}$ is purely normal at $25^{\circ} \mathrm{C}$, while there are regions with both normal and anomalous dispersion at $34.7^{\circ} \mathrm{C}$. The zero dispersion wavelength (ZDW) for this waveguide at $34.7^{\circ} \mathrm{C}$ is at $\lambda=1.58 \mu \mathrm{m}$. The waveguides with radius $r=1.32 \mu \mathrm{m}, 1.35 \mu \mathrm{m}$, and $1.4 \mu \mathrm{m}$ all have ZDWs both at $25^{\circ} \mathrm{C}$ and at $34.7^{\circ} \mathrm{C}$. For the waveguide with radius $1.32 \mu \mathrm{m}$, the ZDW can be tuned in a broad spectrum, spanning from $1.5 \mu \mathrm{m}$ at $34.7^{\circ} \mathrm{C}$ to $1.65 \mu \mathrm{m}$ at $25^{\circ} \mathrm{C}$. The waveguide with $r=1.35 \mu \mathrm{m}$ has a ZDW that can be tuned approximately $30 \mathrm{~nm}$ between $1440 \mathrm{~nm}$ and $1470 \mathrm{~nm}$. The ZDW for the waveguide with $r=1.4 \mu \mathrm{m}$ is at $1440 \mathrm{~nm}$ both at $25^{\circ} \mathrm{C}$ and $34.7^{\circ} \mathrm{C}$. The waveguides considered here are all multimoded at $1550 \mathrm{~nm}$, the single mode cutoff wavelength is between $1.6 \mu \mathrm{m}$ and $2 \mu \mathrm{m}$ for all the possible designs considered. We now use the dispersion curves to find the $\beta$-coefficients in equation (10). In the following we solve equation (10) using pulses with Gaussian shapes as initial conditions. We use a pulse length of $T_{0}=10 \mathrm{ps}$ (FWHM), with a peak power of $P_{0}=1500 \mathrm{~W}$ at $1550 \mathrm{~nm}$, and a repetition rate of $f_{\text {rep }}=100 \mathrm{KHz}$. Since the LC is infiltrated into the tube using capillary forces, long waveguides based on this principle are not possible to produce, therefore we use a length of $10 \mathrm{~cm}$ in the numerical model. In Fig. 3 we have plotted the output spectrum for the waveguide with $r=1.32 \mu \mathrm{m}$ at $25^{\circ} \mathrm{C}$ where the dispersion is normal, and at $34.7^{\circ} \mathrm{C}$ where the dispersion is anomalous. In both cases the broadening of the input spectrum is mainly due to self phase modulation, since the nonlinear effects dominate in both the normal and anomalous dispersion regime. This can shown by introducing a nonlinear length $L_{N L}$ and a dispersion length $L_{D}$, defined by $L_{N L}=\left(\gamma P_{0}\right)^{-1}$ and $L_{D}=T_{0}{ }^{2} / \beta 2 \mid$. Because of the high nonlinearity of the LC and the small value of $\beta_{2}$ near the ZDW, we have that $L_{N}<<L_{D}$. The initial spectrum is about $2 \mathrm{~nm}$ wide, while the generated spectrum is about $100 \mathrm{~nm}$ wide. The output spectrum at $34.7^{\circ} \mathrm{C}$ is also plotted. We see that close to the pump wavelength the two spectra at $25^{\circ} \mathrm{C}$ and $34.7^{\circ} \mathrm{C}$ are similar, but since the dispersion at $34.7^{\circ} \mathrm{C}$ is anomalous, modulational instability is now present, and gives rise to peaks in the 
spectrum far away from the pump wavelength. The position of the peaks can be approximated analytically by considering the parametric gain which is given by [9]

$$
g=\sqrt{-\sum_{j=1}^{\infty} \frac{\beta_{2 j}}{(2 j) !} \Omega^{2 j}\left(2 P_{0} \gamma+\sum_{j=1}^{\infty} \frac{\beta_{2 j}}{(2 j) !} \Omega^{2 j}\right)}
$$

Using the parameters for the waveguide with $r=1.32 \mu \mathrm{m}$ at $34.7^{\circ} \mathrm{C}$, we find that we have maximum gain at $\Omega \approx \pm 233 \mathrm{THz}$, which for a pump wavelength $\lambda_{0}=1550 \mathrm{~nm}$ corresponds to gain peaks at approximately $1300 \mathrm{~nm}$ and $1900 \mathrm{~nm}$. In plot (b) in figure 3 we see that the peaks in the spectrum correspond well to the peaks predicted analytically. Though the intensities of the peaks are much lower than the central peak around the pump wavelength.
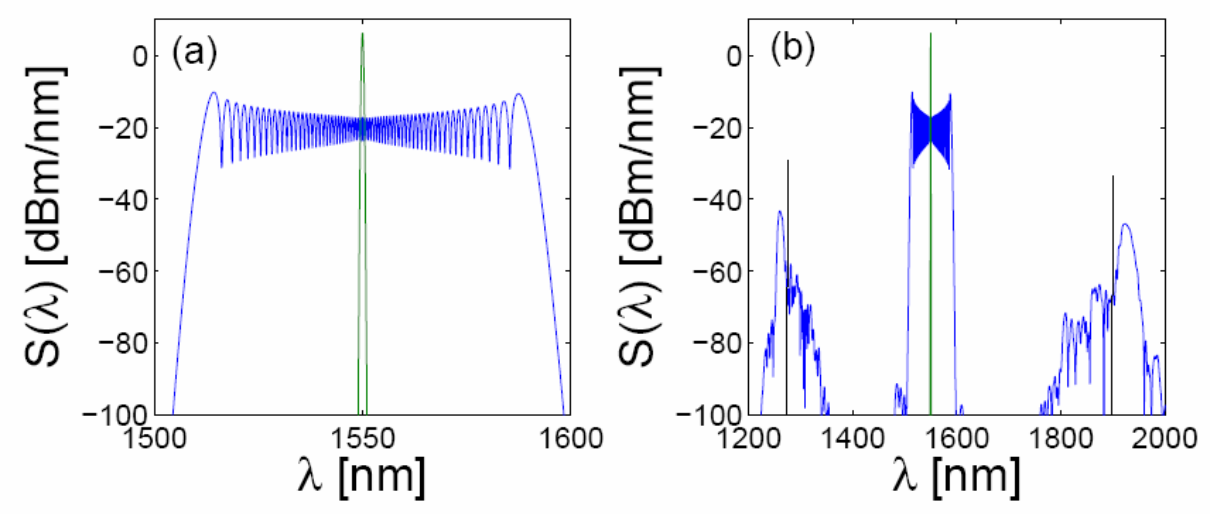

Figure 3. Pump and output spectrum at $25^{\circ} \mathrm{C}$ (a) and at $34.7^{\circ} \mathrm{C}$ (b). The positions of the wavelengths with maximal parametric gain as predicted by equation (12) are marked in (b) with lines at $1300 \mathrm{~nm}$ and at $1900 \mathrm{~nm}$.

\section{CONCLUSION}

We have considered supercontinuum generation in a capillary tube infiltrated with a nematic liquid crystal. Choosing a specific liquid crystal and radius of the capillary tube, we have demonstrated the possibility of supercontinuum generation using $10 \mathrm{ps}$ pulses with peak power $1.5 \mathrm{~kW}$ at $1550 \mathrm{~nm}$. Also we show the possibility of changing the dispersion of the waveguide around $1550 \mathrm{~nm}$ from normal to anomalous by varying the temperature. Using the Nonlinear Schrödinger Equation, output spectra in the normal and anomalous dispersion regime are calculated. Our results show that the broadening of the spectra is mainly due to self phase modulation, since the generated spectra in the normal and anomalous dispersion regime are close to being identical.

\section{REFERENCES}

[1] T.T. Larsen, A. Bjarklev, D.S. Hermann, and J. Broeng: Optical devices based on liquid crystal photonic bandgap fibres, Opt. Exp., vol. 11. pp. 2589 (2003).

[2] N.V. Tabiryan, A. V. Sukhov, and B. Y. Zel'dovich: Orientational Optical Nonlinearity of Lquid Crystals, Mol. Cryst. Liq. Cryst., vol. 136. pp. 1 (1986).

[3] T. Morioka, K. Mori, and M. Saruwatari: More than 100-wavelength-channel picosecond optical pulse generation from single laser source using supercontinuum in optical fibres, Electron. Lett., vol. 29. pp. 862 (1993).

[4] J. Li and S. Wu: Extended Cauchy equations for the refractive indices of liquid crystals, J. Appl. Phys., vol. 95 . pp. 896 (2004).

[5] K. Okamoto, Fundamentals of optical waveguides (Academic Press, San Diego, 2000).

[6] L. Li, H.J. Yuan, G. Hu, and P. Palffy-Muhoray: Third order non-linearities of nematic liquid crystals, Liquid Crystals, vol. 16. pp. 703 (1994).

[7] J.D. Dai and C. K. Jen: Analysis of cladded uniaxial single-crystal fibers, J. Opt. Soc. Am. A, vol. 8. pp. 2021 (1991).

[8] G.P. Agrawal: Nonlinear Fiber Optics (Academic Press, San Diego, 2001).

[9] S. Wen and D. Fan: Spatiotemporal instabilities in nonlinear Kerr media in the presence of arbitrary higher-order dispersions, J. Opt. Soc. Am. B, vol. 19. pp. 1653-1659 (2002). 